\title{
A randomised trial of differentiated prednisolone treatment in active rheumatoid arthritis. Clinical benefits and skeletal side effects
}

\author{
Michael Hansen, Jan Pødenphant, Adrian Florescu, Michael Stoltenberg, Alice Borch, \\ Elga Kluger, Søren Freiesleben Sørensen, Troels Mørk Hansen
}

Department of

Rheumatology,

Hvidovre Hospital,

University of

Copenhagen, Denmark

$M$ Hansen

M Stoltenberg

Department of

Rheumatology and

Radiology, Herlev

Hospital, University of

Copenhagen, Denmark

J Pødenphant

M Stoltenberg

A Borch

T M Hansen

Department of Internal Medicine, TTA Rigshospitalet, National University

Hospital, Denmark

A Florescu

Department of

Rheumatology,

Bispebjerg Hospital,

University of

Copenhagen, Denmark

A Florescu

S F Sørensen

Gråsten Rheumatology

Hospital, Denmark

E Kluger

Correspondence to:

Dr J Pødenphant,

Department of

Rheumatology, Herlev

Hospital.University, of

Copenhagen, Herlev Ringvej,

DK-2730 Herlev, Denmark.

Accepted for publication

26 July 1999

\begin{abstract}
Objectives-To study benefits and skeletal side effects of carefully monitored prednisolone treatment in patients with active rheumatoid arthritis.
\end{abstract}

Methods-One hundred and two patients with active rheumatoid arthritis were randomly allocated to treatment with disease modifying anti-inflammatory drug (DMARD) alone or DMARD and prednisolone in a one year follow up study. Prednisolone was given in a dose regimen adapted to the disease activity of the individual patient. The mean dose was $6 \mathrm{mg}$ and the mean cumulated dose was 2160 mg. Patients were followed up with disease activity parameters, radiograph of the hands (Larsen score), and bone mineral density (BMD) of the lumbar spine, distal forearm and hand. At one year 26 patients had withdrawn from the investigation leaving 76 patients for evaluation.

Results-The results showed that disease activity in the prednisolone treated group was reduced within two weeks. In the DMARD alone group disease activity was gradually reduced over months. At six months there was no difference between the groups as evaluated by an improvement score using a number of ACR criteria. Prednisolone in the present set up was not able to protect significantly against radiological disease progression, although there was a trend towards less progression in Larsen score in the prednisolone group, a matter that was further underlined in an intention to treat analysis. BMD data revealed a significant reduction in spinal BMD in the prednisolone group, whereas prednisolone seemed to have a protective effect against bone loss in the hand and distal forearm.

Conclusions-This study does not allow any firm conclusions for or against the treatment of rheumatoid arthritis with prednisolone. The data suggest that the beneficial effects of prednisolone are not as clear cut in established rheumatoid arthritis as in early disease. Furthermore the data indicate that treatment in the chosen relatively low dose does not provide sufficient control of disease. On the other hand the spinal bone loss observed in the prednisolone group does invite considerations about using higher doses.

(Ann Rheum Dis 1999;58:713-718)
Rheumatoid arthritis is a chronic progressive disease. The current concept of treatment is early intervention with disease modifying antirheumatic drugs (DMARD) to arrest joint destruction, which often progresses rapidly in early disease. ${ }^{1}$ Unfortunately, there is a considerable time span between the initiation of DMARD treatment and its effect on disease activity usually three to six months. To ameliorate the patient's symptoms treatment with a corticosteroid in an adequate dose may be considered to control the disease activity. Prednisolone is able to suppress clinical and biochemical disease activity and a recent study has shown that it has a protective effect on joint destruction. ${ }^{2}$ Prednisolone, however, has a number of side effects. One of the most serious is bone loss leading to fractures and disability. ${ }^{3}$

In patients with rheumatoid arthritis, the disease itself seems to predispose to bone loss and there is evidence that this loss is related to disease activity. ${ }^{3-5}$ Accordingly one study of early rheumatoid arthritis in which a small subgroup of patients received prednisolone, suggests that prednisolone has a beneficial effect on bone loss.

In this study we randomised 102 patients with active rheumatoid arthritis to treatment with prednisolone in a dose presumed to be sufficient to control their disease activity or no prednisolone. All patients received DMARD treatment and all were recruited at a time when active disease made it necessary either to start or change DMARD treatment. The aim of the investigation was to study the benefits and skeletal side effects of carefully monitored prednisolone treatment in patients with active disease.

\section{Methods}

\section{STUDY DESIGN}

The design was a longitudinal multicentre study with five centres. One hundred and two patients with active rheumatoid arthritis according to ARA criteria ${ }^{6}$ and who planned either to start or change DMARD treatment were randomly allocated to treatment with or without prednisolone. Distribution of patients among the various centres were $40,12,20,10$, 20. Randomisation was done as block randomisation with 10 patients per block. The randomisation procedure was carried out at the centre contributing 40 patients (Hvidovre Hospital). Each centre received blocks as needed. The criteria for active disease were more than three swollen joints and two of the 


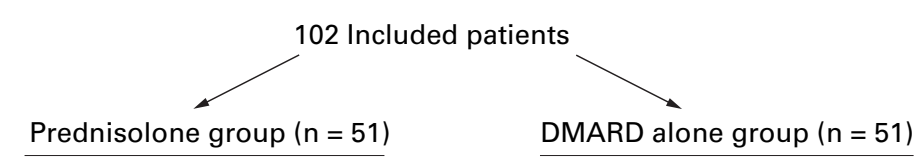

Withdrawn because of:
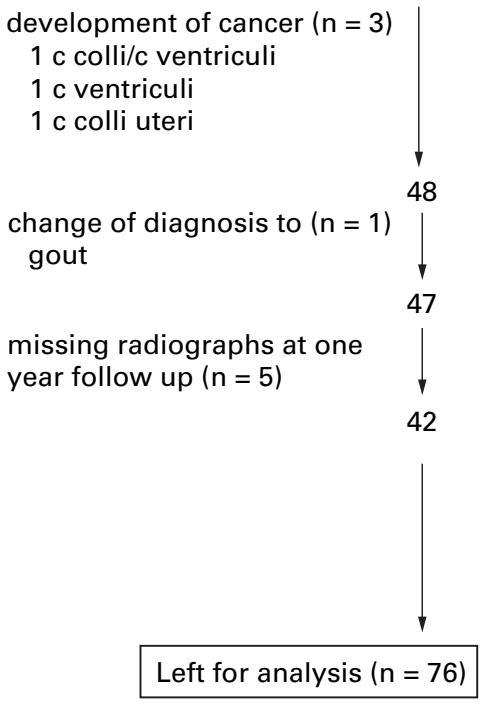

\section{development of cancer $(n=4)$} $1 \mathrm{c}$ ovarii

$1 \mathrm{c}$ colli uteri

$1 \mathrm{c}$ pulmones

$1 \mathrm{c}$ colli uteri

47

change of diagnosis to $(n=1)$ psoriatrica artritis

46

missing radiographs at one year follow up $(n=7)$

39

treatment failure: + prednisolone $(n=4)$

35

non-compliance $(n=1)$

Figure 1 A CONSORT flow diagram to show patient courses during the study.

three following criteria, morning stiffness over 30 minutes, erythrocyte sedimentation rate $(\mathrm{ESR})>35 \mathrm{~mm} 1 \mathrm{st} \mathrm{h}$, and $\mathrm{C}$ reactive protein $(\mathrm{CRP})>150 \mathrm{nmol} / \mathrm{l}$. Patients with metabolic bone disease, liver disease, diabetes mellitus, malignant disease, other connective tissue disease, patients in Steinbrocker class IV, and patients who had received systemic corticosteroids within the preceding six months were not included in the study. Twenty six patients withdrew from the study during the one year investigation. Figure 1 shows the data. This left 42 patients $(32 \mathrm{~F} / 10 \mathrm{M})$ in the prednisolone treated group and 34 patients $(26 \mathrm{~F} / 8 \mathrm{M})$ in the DMARD only group for evaluation. An intention to treat analysis on available radiographic data was done in 82 patients. These included 43 patients in the prednisolone treated group and 39 patients in the DMARD only group. The additional patients were one with compliance problems (prednisolone group), three newly diagnosed malignancies, one treatment failure and one with compliance problems (DMARD only) group.

Prednisolone treatment was given as $30 \mathrm{mg}$ once a day the in first week, $20 \mathrm{mg}$ once a day in the second week and $15 \mathrm{mg}$ once a day, day 15. Thereafter the patients were asked to choose a prednisolone dose between 2.5 and $15 \mathrm{mg}$, which would be sufficient to control their disease activity. They were given information on the clinical signs of active diseasetender joints and morning stiffness. The patients were allowed to change the daily prednisolone dose by $1.25 \mathrm{mg}$, at a time. The study ran for one year, and the individual patient was followed up by the same rheumatologist during this period. All patients were informed verbally and in writing in accordance with the Helsinki 11 declaration, and the study was approved by the local ethical committees of the participating centres.

\section{CONCURRENT TREATMENT}

NSAIDs and simple analgesics were permitted. In the DMARD plus prednisolone group 34 of 42 patients took NSAIDs at the start of the study and 28 of 42 at the end of the study (one year). In the DMARD only group, the numbers were 28 of 34 at the start and 25 of 34 after one year. Twenty two of the patients in each treatment group were postmenopausal women. Four in the DMARD plus prednisolone and one in the DMARD only group received hormone replacement therapy throughout the study. Calcium and vitamin D was given to two patients at study start and three patients after one year in the DMARD + prednisolone group. No patients in the DMARD alone group received calcium supplementation. Intra-articular corticosteroid (Depomedrol $20-80 \mathrm{mg}$ ) was given to 10 patients, total 13 injections in the DMARD + prednisolone group and 11 patients, total 12 injections in the DMARD alone group.

\section{CLINICAL VARIABLES}

Number of swollen and tender joints, a health assessment questionaire (HAQ-score), ${ }^{7}$ patients' and doctors' global evaluation according to an 11 point box scale ranging from 0 , best possible to 10 , worst possible and the grip strength ${ }^{5}$ were recorded at the start of the study at one and two weeks, thereafter once a month for the subsequent six months and at one year. Tender and swollen joints and at least two of the following three variables, doctors' global evaluation, HAQ score and CRP were used in an improvement score to test $20 \%$ and $50 \%$ improvement.

\section{RADIOGRAPHS}

Radiographs of hands and wrists were taken at the time of entry and one year later. All radiographs were evaluated by the same experienced 
Table 1 Clinical and paraclinical data of the two groups at the time of entry. Data are given as median and range

\begin{tabular}{|c|c|c|c|}
\hline & $\begin{array}{l}\text { DMARD alone } \\
(n=34)\end{array}$ & Prednisolone $(n=42)$ & Significance \\
\hline Age (y) & $65(26-80)$ & $60(20-83)$ & NS \\
\hline Disease duration (y) & $8.5(0.5-36)$ & $2.8(0.5-30)$ & $\mathrm{p}<0.05$ \\
\hline Steinbrocker class 1 / 11 / 111 & $20 / 13 / 1$ & $24 / 18 / 0$ & \\
\hline Tender joints $(\max 40)$ & $7(0-38)$ & $8(0-37)$ & NS \\
\hline Swollen joints (max 40) & $9(3-29)$ & $12(5-24)$ & NS \\
\hline Doctors' global (0-10) & $6(2-8)$ & $6(3-10)$ & NS \\
\hline Patients' global (0-10) & $6(2-10)$ & $7(3-10)$ & NS \\
\hline HAQ score $(0-3)$ & $0.9(0-2.3)$ & $1.0(0.1-1.8)$ & NS \\
\hline $\operatorname{ESR}(\mathrm{mm} \mathrm{1st} \mathrm{h)}$ & $44(10-97)$ & $45(12-110)$ & NS \\
\hline $\mathrm{CRP}(\mathrm{nmol} / \mathrm{l})$ & $348(29-988)$ & $310(35-1771)$ & N.S \\
\hline Larsen score & $31.5(15-45)$ & $28.5(12-37)$ & NS \\
\hline $\mathrm{BMD}$ spine $\left(\mathrm{g} / \mathrm{cm}^{2}\right)$ & $0.867(0.732-1.030)$ & $0.987(0.0 .909-1.075)$ & $\mathrm{p}<0.01$ \\
\hline $\mathrm{BMD}$ arm $\left(\mathrm{g} / \mathrm{cm}^{2}\right)$ & $0.372(0.304-0.457)$ & $0.413(0.354-0.451)$ & NS \\
\hline $\mathrm{BMD}$ hand $\left(\mathrm{g} / \mathrm{cm}^{2}\right)$ & $0.222(0.166-0.272)$ & $0.220(0.196-0.257)$ & NS \\
\hline
\end{tabular}

radiologist $(\mathrm{AB})$ in randomly ordered groups. Any markings that could identify patients were covered. The evaluation was done according to the method of Larsen et al, ${ }^{8}$ which grades joint damage on a scale from 0 , no damage to 5 , maximum damage.

BONE MINERAL DENSITY

$\mathrm{BMD}$ of the lumbar spine, non-dominant distal forearm and hand was measured by a Norland XR-26 DEXA scanner at the start of the study and at one year. The small animal programme on the scanner was used for the hand measurements. The scanner operates by an $x$ ray principle with high precision and accuracy. Further details are given elsewhere. ${ }^{59}$ The technician operating the scanner was unaware of the treatment regimens and scanning results were not analysed until the end of the trial.

\section{BIOCHEMISTRY}

ESR and CRP were measured by the same standard procedures in the laboratories of the participating hospitals at the same time intervals as for the clinical variables. Biochemical variables to exclude certain other diseases (see study design) were measured at the start of the study and safety variables to monitor DMARD treatment were measured at intervals decided by the rheumatologists responsible.

\section{STATISTICS}

The data in table 1 are given as median and range. Comparison between groups were calculated by the non-parametric MannWhitney test for unpaired differences and by Wilcoxon's test for comparison within groups. To compare the bone mass measurements in the patients with normal age and sex matched levels the standard deviation ( $Z$ scores) were calculated by the equation: (patients' measurement minus mean for age and sex matched healthy subjects divided by standard deviation for age and sex matched healthy subjects. Differences between patients and controls were calculated by one way analysis of variance.

To estimate the longitudinal changes in disease activity (improvement score) and changes in $\mathrm{BMD}$ during treatment the initial values were set to $100 \%$ and values obtained after institution of treatment were expressed as percentage of the initial value. Differences be-
Table 2 DMARD treatment in the two groups

\begin{tabular}{lll}
\hline & $\begin{array}{l}\text { DMARD } \\
\text { prednisolone }\end{array}$ & $\begin{array}{l}\text { DMARD } \\
\text { alone }\end{array}$ \\
\hline Auronofin & 2 & 0 \\
Aurothiomalat & 1 & 4 \\
Azathioprine & 1 & 0 \\
Hydroxychlorokine & 1 & 1 \\
Methotrexate & 19 & 15 \\
Penicillamine & 5 & 1 \\
Sulfasalazine & 13 & 13 \\
\hline
\end{tabular}

Table 3 Rates of change in bone mineral density (BMD) at various sites in the two treatment groups. Data are given as mean change with $95 \%$ confidence intervals

\begin{tabular}{lll}
\hline & $\begin{array}{l}\text { DMARD + } \\
\text { prednisolone }(n=42)\end{array}$ & $\begin{array}{l}\text { DMARD only } \\
(n=34)\end{array}$ \\
\hline Delta BMD spine & $-2.7(-4.5,-0.9)$ & $-1.0(-3.2,+1.2)$ \\
Delta BMD arm & $-1.6(-3.7,+0.5)$ & $-4.2(-6.6,-1.9)$ \\
Delta BMD hand & $0.9(-2.3,+4.1)$ & $-3.7(-6.4,-0.9)$ \\
\hline
\end{tabular}

tween the percentage change in improvement score and BMD data between the two treatment groups were estimated by Student's $t$ test for unpaired data.

Differences between groups for the absolute values of radiographic progression and improvement score were tested by $2 \times 2$ tables. Multiple regression analysis was used to test if disease duration had an effect on BMD at start of study or changes in BMD. $p$ Values less than or equal to 0.05 were considered significant.

\section{Results}

Table 1 gives the clinical data of the 76 patients at the time of entry into the study. It seems that by chance disease duration was longer in the DMARD alone group compared with the prednisolone group, and that BMD spine was significantly lower in the DMARD only group, also when corrected for sex and age. Data analysis was unable to account for this coincidence. The mean daily prednisolone dose during the year of treatment was $6 \mathrm{mg}$ and the mean cumulated dose was $2160 \mathrm{mg}$. At six months the mean dose was $4.5 \mathrm{mg}$ and at one year $3.0 \mathrm{mg}$. In table 2 types of DMARDs in the two treatment groups is shown. It seems, that the groups are comparable with most patients in treatment with methotrexate or sulfasalazine.

Radiographic analysis (Larsen score) showed a significant progression in joint destruction in the DMARD only group from 34.3 to 37.7 whereas the score of the prednisolone group progressed from 29.4 to 31.2. In both groups the progression was highly significant $(p<0.01)$. Comparison of the rate of progression in the two groups (delta Larsen score) 3.4 in the DMARD alone group versus 1.8 in the DMARD + prednisolone group was non-significant $(p<0.07)$, and comparison of number of patients progressing in the two groups gave 22 of $34(65 \%)$ in the DMARD alone group and 20 of $42(48 \%)$ in the DMARD + prednisolone group $(\mathrm{p}<0.14$, nonsignificant). In 82 patients, 39 in the DMARD alone and 43 in the DMARD + prednisolone group, it was possible to make an intention to treat analysis of Larsen score. This analysis revealed a significantly higher rate of 

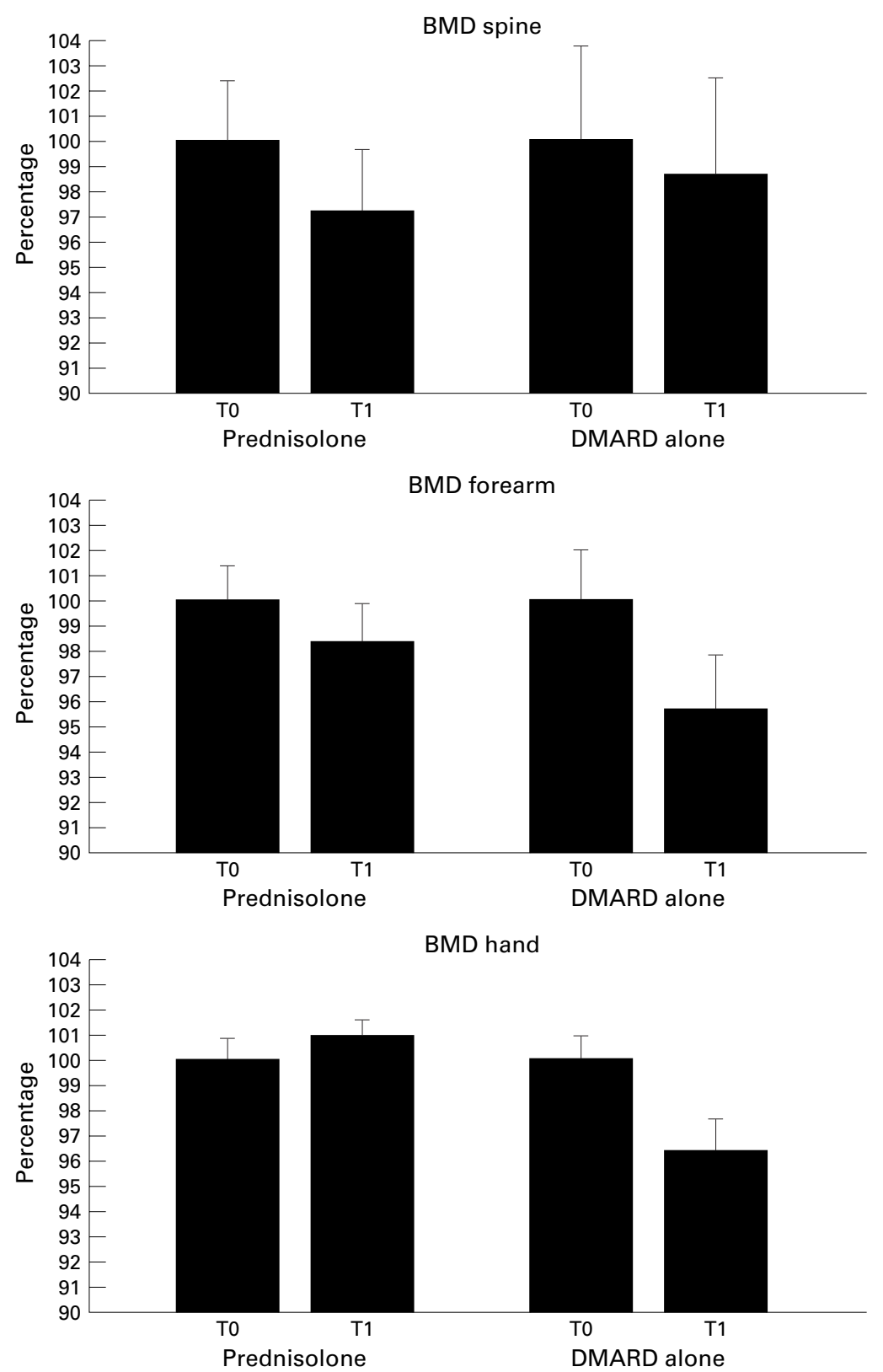

Figure 2 Changes in bone mineral density (BMD) of the lumbar spine, distal forearm, and hand during one year of treatment with DMARD alone or DMARD + prednisolone. The values at T-start are set at 100\% and values at T-1 year are given in per cent of the starting value. Bars indicate standard error of the mean (SEM \%).

progression (delta Larsen score) in the group on DMARD alone 3.5 versus $1.8(\mathrm{p}<0.03)$. Comparison of number of patients progressing in the two groups gave 25 of $39(64 \%)$ in the DMARD alone group and 20 of $43(47 \%)$ in the DMARD + prednisolone group $(\mathrm{p}<0.07$, non-significant).

Figure 2 shows the bone mineral density in the two groups at the start and at one year. The BMD value at the start was set at $100 \%$ and that at one year is given in per cent of the starting value. A significant reduction in BMD spine of $2.7 \%(p<0.05)$ was found in the prednisolone group, whereas the DMARD only group remained by and large unchanged. In contrast the distal forearm and hand show a quite different result. In these areas, the patients taking prednisolone had almost the same BMD values at one year, whereas the patients taking DMARD alone showed a significant reduction in both BMD forearm
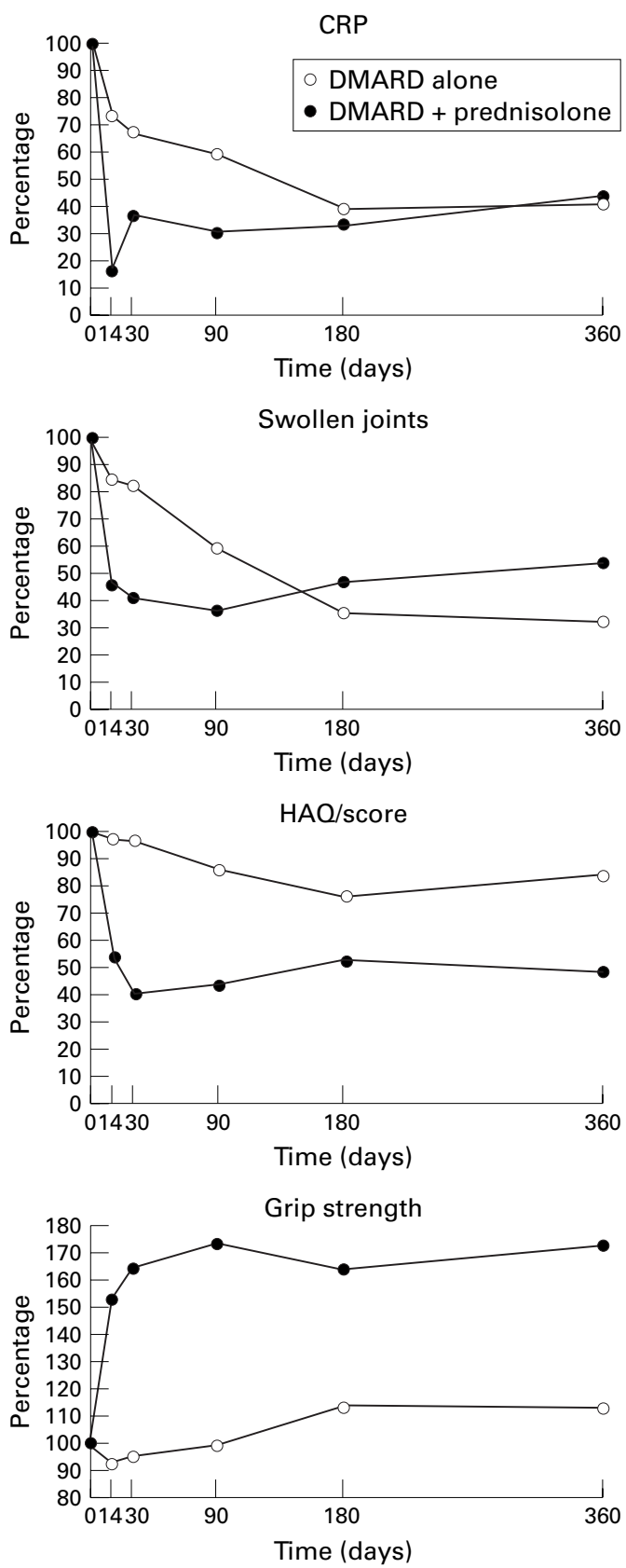

Figure 3 Changes in clinical and biochemical variables of disease activity during one year of treatment with DMARD alone or DMARD + prednisolone. $H A Q=$ Health

Assessment Questionnaire.CRP $=C$ reactive protein. Data are shown as mean \% of T-start.

and BMD hand at one year, $4.2 \%$ and $3.7 \%$ respectively $(p<0.05)$. Furthermore, a significant difference in delta BMD hand between the groups was found $(\mathrm{p}<0.05)$. In the calculation of reduction in BMD hand in the DMARD alone group we excluded an outlier that improved 53\% during the study. Data are further detailed in table 3 . As data at T0 were not comparable for length of disease and $\mathrm{BMD}$ we did a multiple regression analysis to establish whether duration of disease had an effect on BMD Z scores in the regions of interest. Only treatment with glucocorticoid had a significant influence on changes in BMD.

Figure 3 depicts the changes in the activity parameters-number of swollen joints, CRP, HAQ score and grip strength over the year. Disease activity seems to be brought under control in the prednisolone treated group 
Table 4 The 20\% and 50\% improvement score at various time intervals (days) T0 = immediate improvement (within two weeks). Data are given as $+1-$ improvement $>20 \%$ (a) and $>50 \%$ (b)

\begin{tabular}{|c|c|c|c|c|c|c|}
\hline & \multicolumn{2}{|c|}{ Prednisolone + DMARD } & \multicolumn{2}{|l|}{$D M A R D$ alone } & \multicolumn{2}{|c|}{ Significance } \\
\hline & $a$ & $b$ & $a$ & $b$ & $a$ & $b$ \\
\hline Т0 & $26 / 42(61.9 \%)$ & $17 / 42(40.4 \%)$ & $6 / 34(17.6 \%)$ & $0 / 34(0 \%)$ & $\mathrm{p}<0.001$ & $\mathrm{p}<0.001$ \\
\hline T90 & $24 / 42(57.1 \%)$ & $14 / 42(33.3 \%)$ & $10 / 34(29.4 \%)$ & $0 / 34(0 \%)$ & $\mathrm{p}<0.02$ & $\mathrm{p}<0.001$ \\
\hline $\mathrm{T} 180$ & $22 / 42(52.4 \%)$ & $13 / 42(31.0 \%)$ & $17 / 34(50 \%)$ & $12 / 34(35.3 \%)$ & NS & NS \\
\hline T360 & $16 / 42(38.1 \%)$ & $8 / 42(19 \%)$ & $14 / 34(41.2 \%)$ & $10 / 34(29.4 \%)$ & NS & NS \\
\hline
\end{tabular}

within the first two weeks with a reduction in the activity parameters of $50 \%$ or more. At 180 days there was no significant difference between the groups in the number of swollen joints and CRP. A positive effect on the HAQ score and grip strength persisted throughout the study. Table 4 gives a clinical improvement score showing a minimum improvement of $20 \%$ and $50 \%$ in tender and swollen joints, and $20 \%$ and $50 \%$ improvement in at least two of the following variables, doctors' global assessment, HAQ score, and CRP. The variables used were available data chosen to match the ACR criteria for improvement as closely as possible. ${ }^{10}$ The results show an initial improvement in the DMARD plus prednisolone group, but at six months there was no difference between the groups.

\section{Discussion}

Prednisolone is a drug often prescribed for patients with rheumatoid arthritis. In their recent study Kirwan et al produced convincing data that in a fixed dose of $7.5 \mathrm{mg}$ to patients with early disease, prednisolone was indeed able to delay joint destruction, however, they did not examine the side effects on mineralised bone. Another study of early rheumatoid arthritis by Boers et $a l^{11}$ also showed a reduction in joint destruction in a group of patients treated with sulfasalazine, methotrexate and prednisolone compared with sulfasalazine alone. In that study prednisolone was given in a starting dose of $60 \mathrm{mg}$, which was tapered to a dose of $7.5 \mathrm{mg}$ over the course of six weeks. Prednisolone treatment was stopped at 28 weeks. The cumulative dose corresponds to that given in this study. The clinical results also compare well with our findings-an initial fast reduction in disease activity in the prednisolone treated group, but no difference at 28 weeks when prednisolone was stopped. Similiarly in our study, where the mean dose of prednisolone was $4.5 \mathrm{mg}$ a day the clinical improvement score showed no difference at 26 weeks (180 days). There is, however, one substantial difference between our study and the Boers study. Whereas the latter did not show any significant difference between the treatment groups in the BMD of the lumbar spine, our study showed a significant reduction in the prednisolone group. On the other hand BMD was not lost in the hand or distal forearm, as was found in the patients receiving DMARD alone. The data suggest that the deleterious effect of prednisolone on bone in these areas is counterbalanced by a beneficial effect on the rheumatoid inflammation, which in itself is known to cause bone loss. ${ }^{4}$ Lumbar bone mineral density data from another randomised longitudinal study with a patient group and prednisolone dose comparable to the present, however, also showed significant but reversible bone loss in the corticosteroid group. ${ }^{12}$

Our study differs from the first two above mentioned investigations in that we did not find a protective effect on joint destruction, although there was a trend in favour of the prednisolone group. This trend was further underlined by significant differences in rate of progression in an intention to treat analysis. An obvious explanation is that our patients were not an early rheumatoid arthritis group but an unselected patient group with varying duration of disease. A possible pitfall is that the patients in the DMARD only group in the present set up by chance had a significantly longer duration of disease and a higher initial Larsen score than had the patients in the prednisolone group. This circumstance may reduce the degree of the progression of the disease, as evaluated by the Larsen score. There is thus the possibility that a significant reduction in progression occurred in the prednisolone group, which was missed.

Another important point is that the full impact on prevention of disease progression may require a higher level of disease control than obtained with the present dose regimen. Thus the results of two classic studies ${ }^{13} 14$ showed a reduction in the radiological disease progression in patients, who received two years of high dose treatment with prednisolone, whereas little influence was seen during the third year, when the dose was low-about 10 mg.

Our study does not permit any firm conclusion for or against the treatment of rheumatoid arthritis with prednisolone. The data suggest that the beneficial effects of prednisolone are not as clear cut in established disease as in early rheumatoid arthritis. The data furthermore indicate that treatment in the chosen relatively low dose (mean $6 \mathrm{mg}$ ) does not provide sufficient control of disease. On the other hand the spinal bone loss observed in the prednisolone group does invite considerations about higher doses. One may, however, anticipate that newer bone sparing agents such as the bisfosfonates may manage this problem.

Funding: this study was supported by grants from "The Danish Rheumatism Association" and "The Velux Foundation of 1981 ”.

We thank the doctors in the participating centres: Gert Overeem Hansen, Kim Hørslev-Petersen, Ib Lorenzen, Vagn Andersen, Gunnar Bendixen, and nurse Birgitte Pedersen-Zbinden.

1 Salaffi F, Ferraccioli G, Peroni M, Carotti M, Bartoli E, Cervini C.Progression of erosion and joint space narrowing scores in rheumatoid arthritis assessed by nonlinear models. J Rheumatol 1994;21:1626-30. 
2 Kirwan JR. The effect of glucocorticosteroids on bone destruction in rheumatoid arthritis. $\mathrm{N}$ Engl J Med 1995;333:142-6

3 Hall GM, Spector TD, Griffin AJ, Jawad ASM, Hall ML Doyle DV. The effect of rheumatoid arthritis and steroid therapy on bone density in postmenopausal women. Arthritis Rheum 1993;36:1510-16.

4 Gough AKS, Lilley J, Eyre S, Holder RL, Emery P. Generalised bone loss in patients with early reumatoid arthritis. Lancet 1994;344:23-7.

5 Hansen M, Florescu A, Stoltenberg M, Pødenphant J, Pedersen-Zbinden B, Hørslev-Petersen K, et al. Bone loss in rheumatoid arthritis. Scand J Rheumatol 1996;23:367in rh.

6 Arnett FC, Edsworthy SM, Bloch DA, McShane D, Fries JF, Cooper NS, et al. The American Rheumatism AssociaJF, Cooper NS, et al. The American Rheumatism Associa-
tion 1987 revised criteria for the classification of rheumation 1987 revised criteria for the classification

7 Fries JF, Spitz PW, Young DY. The dimensions of health outcomes. The health assessment questionaire, disability and pain scales. J Rheumatol 1982;9: 789-93.

8 Larsen A, Dale K, Eek M. Radiographic evaluation of rheumatoid arthritis and related conditions by standard reference films. Acta Radiol Diag 1977;18:481-91.

9 Lilley J, Walters BG, Heath DA, Drole Z. In vivo and in vitro precision for bone density measured by dual energy x-ray absorption. Osteoporosis Int 1991;1:141-6.
10 Felson DT, Anderson JJ, Boers M, Bombardier C, Furst D, Kluger E, et al. American College of Rheumatology preliminary definitions of improvement in rheumatoid arthritis. Arthritis Rheum 1995;38:727-35.

11 Boers M, Verhoeven AC, Markusse HM, van de Laar MAFI, Westhoevens R, et al. Randomised comparison of combined step-down prednisolone, methotrexate and combined step-down sulphasalazine with sulphasalazine alone in early rheumatoid arthritis. Lancet 1997;350:309-18.

12 Laan RFJM, van Riel PLCM, van de Putte LBA, van Erning LJTh O, van't Hof MA, Lemmers AM. Low-dose prednisolone induces rapid reversible axial bone loss in patients with rheumatoid arthritis. Ann Intern Med 1993;10:963-8.

13 Report by the joint committee of the Medical Research Council and Nuffield Foundation on clinical trials of cortisone, ACTH, and other therapeutic measures in chronic rheumatic diseases. A comparison of prednisolone with reumatic diseases. A comparison of prednisolone with aspirin or other analgesics in the treatment

14 A second report by the joint committee of the Medical Research Council and Nuffield Foundation on clinical trials of cortisone, ACTH, and other therapeutic measures in chronic rheumatic diseases. A comparison of prednisolone with aspirin or other analgesics in the treatment of rheumatoid arthritis. Ann Rheum Dis 1960;19:331-7. 